\title{
Correlation and Path Co-efficient Analysis in Ridge Gourd [Luffa acutangula (L.) Roxb.]
}

\author{
K. Hanume Gowda* \\ ICAR-CTCRI, Regional Centre, Dumuduma Housing Board, \\ Bhubaneswar-751019, Odisha, India \\ *Corresponding author
}

\section{A B S T R A C T}

\section{Keywords}

Ridge gourd, Correlation, Path co-efficient analysis, Fruit yield per plant

\section{Article Info}

Accepted:

22 June 2020

Available Online:

10 July 2020
Correlation and path co-efficient analysis for 18 traits were conducted for thirty genotypes of ridge gourd during 2010-11 at Kittur Rani Channamma College of Horticulture, Arabhavi, Karnataka, India. The Values of genotypic correlation coefficient were greater than the phenotypic correlation co-efficient. Fruit yield per plant was highly significant and positively correlated with vine length at 45 and 90 DAS, number of branches at 90 DAS, number of leaves at 45 and 90 DAS, node to first female and male flowering, days to last harvest, sex ratio, number of fruits per plant, fruit yield per plot, average fruit weight and fruit length at both genotypic and phenotypic level. Whereas days to first female flowering, days to 50 per cent flowering, days to first male flowering and days to first harvest were negative and highly significant correlation with fruit yield per plant. Path coefficient analysis revealed that number of fruits per plant had the direct effect on yield, followed by average fruit weight and fruit length both at phenotypic and genotypic level. Thus, selection for these characters will improve the yield. The results indicated that number of fruits per plant and average fruit weight can be considered during selection for improved yield in ridge gourd.

\section{Introduction}

Ridge gourd [Luffa acutangula (L.) Roxb.] is an important warm season vegetable grown all over India and having long history of cultivation in the tropical countries of Asia and Africa (Sheshadri, 1980). Ridge gourd belongs to genus Luffa of Cucurbitaceae family and has chromosome number $2 \mathrm{n}=26$.
Ridge gourd originated in India, it is cultivated in the tropics for its tender edible fruits both on commercial scale and in kitchen gardens throughout India and it is popular vegetable both as spring summer and rainy season crop. Seeds are reported to be possess purgative, emetic and antihelmintic properties due to the secondary metabolite cucurbitacin (Robinson and Decker-Walters, 1997). 
Ridge gourd is large climber with long tap root system and leaves green, simple and ovate with 5-7 lobes. Though cultivated species are monoecious in nature different sex forms viz., androecious, gynoecious, gynomonoecious, andromonoecious and hermaphrodite forms are also reported (Choudhary and Thakur, 1965). The staminate flowers with 5 stamens (synandry) are born in 10-20 flowered racemes while pistillate flowers are solitary, short or long pedunculate and fragrant. Pistillate and staminate flowers are borne on axil of leaf. Anthesis starts between 4 to $6 \mathrm{pm}$ in the evening and remain throughout the night and are ready for selfing and pollination in the early morning or afternoon. Anthesis and dehiscence are governed by temperature and humidity. Anthers are free and pistil has three placenta with many ovules. Stigmas are three and bilobate. Fruit is club shaped and angled with ten prominent ridges and many seeded. It becomes hard, ridged and inedible on maturity. Seeds are black, flattened and wrinkled. One thousand seeds weigh about 150 to $170 \mathrm{~g}$ (Doijode, 2002).

The crop improvement work should be focused on selection of genotypes for better yield, superior quality and resistance to biotic stresses. Selection is an intrinsic part of all vegetable crop improvement programmes and it is as old as cultivation itself. For an effective selection, information on the nature and magnitude of variation available in the material with regard to component characters contributing to yield and the part played by the environment in the expression of these plant characters is essential. In selecting a plant or a type, one should be reasonably sure of the superiority of the selection being inherited by the progenies. This is because a sizable part of the phenotypic variation is caused by environmental factors. The biometrical methods applied in crop improvement programme provide means of evaluating the phenotypic expression of characters in terms of their genotypic worth.

Before aiming an improvement in yield, it is necessary to have the knowledge of correlation and path co-efficient analysis. However, selection based on yield alone is often misleading, because it is one of the most complex character being dependent on its components for its full expression. For rational improvement of yield and its components, association of component characters with yield and among the components themselves should be found out by estimating the correlation co-efficient.

Association of characters determined by correlation co-efficient, although useful will not provide an exact picture of the relative importance of direct and indirect influence of each of the characters towards yield. Path coefficient analysis was developed by Wright (1921) has been employed in many vegetables in order to overcome the unreliability of correlation co-efficient, this technique involves effective partitioning of the correlation co-efficient in to measures of direct and indirect effects on yield. Much work has not been done for its genetic improvement of ridge gourd. Hence an investigation was undertaken to study the correlation and path co-efficient analysis in ridge gourd.

\section{Materials and Methods}

The study was conducted with thirty genotypes of ridge gourd in Randomized Complete Block Design (RCBD) with two replication at Department of Vegetable Science, Kittur Rani Channamma College of Horticulture, Arabhavi during 2010-11 (August-2010 to January-2011). The spacing maintained between rows was $1.2 \mathrm{~m}$ and between plants was $0.9 \mathrm{~m}$. Irrigation, weed control and other cultural practices were 
followed as per the package of practices of horticultural crops of University of Agricultural Sciences, Dharwad (Anon., 2008). The vines were staked individually using wooden sticks and were trained uniformly. This helped the plants for better spreading and easy harvesting. Observations were recorded on growth, earliness, yield and yield contributing characters to know correlation of characters with yield.

The correlation co-efficient among all possible character combinations at phenotypic (rp) and genotypic (rg) level were estimated employing formula (Al-Jibouri et al., 1958). The correlation co-efficient among all possible character combinations at phenotypic (rp) and genotypic (rg) level were estimated employing formula (Al-Jibouri et al., 1958).

Phenotypic correlation $=r_{x y}(p)=$

$$
\frac{\operatorname{Cov}_{x y}(p)}{\sqrt{V_{x}(p) \times V_{y}(p)}}
$$

Genotypic correlation $=r_{x y}(g)=$

$$
\frac{\operatorname{Cov}_{x y}(g)}{\sqrt{V_{x}(g) \times V_{y}(g)}}
$$

Where,

$\operatorname{Cov}_{x y}(G)=$ Genotypic covariance between $x$ and $y$

$\operatorname{Cov}_{\mathrm{xy}}(\mathrm{P})=$ Phenotypic covariance between $\mathrm{x}$ and $\mathrm{y}$

$\mathrm{V}_{\mathrm{x}}(\mathrm{G})=$ Genotypic variance of character ' $\mathrm{x}$ '

$V_{x}(P)=$ Phenotypic variance of character ' $x$ '

$V_{y}(G)=$ Genotypic variance of character ' $y$ '

$V_{y}(P)=$ Phenotypic variance of character ' $y$ '

The test of significance for association between characters was done by comparing table ' $r$ ' values at $n-2$ error degrees of freedom for phenotypic and genotypic correlations with estimated values, respectively.

Path co-efficient analysis suggested by Wright (1921) and Dewey and Lu (1957) was carried out to know the direct and indirect effect of the morphological traits on plant yield.

\section{Results and Discussion}

The genotypic and phenotypic correlation coefficient were determined to obtain information on the relationship among all 18 quantitative characters in ridge gourd and are presented in Tables 2 and 3, respectively.

Analysis of variance showed significant differences for all the characters under study (Table-1). Correlation co-efficient analysis revealed that fruit yield per plant showed positive and significant correlation with number of fruits per plant (0.889), average fruit weight (0.826), fruit yield per plot (0.821), fruit length $(0.795)$, number of leaves at 90 days after sowing (0.795), vine length at 90 days after sowing (0.774), days to last harvest (0.717), number of branches at 90 days after sowing (0.708), number of leaves at 45 days after sowing (0.692) and vine length 45 days after sowing (0.616) at genotypic level. Whereas number of fruits per plant (0.862), average fruit weight $(0.858)$, fruit yield per plot (0.807), fruit length (0.784), number of leaves at 90 days after sowing (0.768), vine length at 90 days after sowing (0.760), days to last harvest (0.682), number of branches 90 days after sowing (0.650), number of leaves at 45 days after sowing (0.599) and vine length 45 days after sowing (0.517) at phenotypic level. Since, these association characters are in the desirable direction, selection for these traits may improve the yield per plant. These results are in confirmed with Varalakshmi et al., (1995), Rao et al., (2000), Chowdhury and Sarma 
(2002) and Prasanna et al., (2002) in ridge gourd. Fruit yield per plant was highly significant and negative association with days to first female and male flowering (-0.690 and -0.611 ), days to 50 per cent flowering ($0.684)$ and days to first harvest (-0.612) indicating that earliness parameters were negatively correlated with fruit yield, which adversely affect the fruit yield.

Path co-efficient analysis determined to obtain information on the direct and indirect effect of characters on fruit yield per plant among all 18 quantitative characters in ridge gourd and are presented in Tables 4 and 5, respectively.

Path co-efficient analysis shows that the number of branches at 90 days after sowing, number of leaves at 90 days after sowing, number of fruits per plant, average fruit weight, fruit yield per plot and days to last harvest had direct positive effect on fruit yield. Among these number of fruits per plant, average fruit weight and fruit yield per plot had high direct positive effect on fruit yield indicating their true positive and significant association with yield.

Table.1 Analysis of variance (mean sum of squares) for growth, earliness, yield, fruit quality and seed parameters in Ridge gourd (Luffa acutangula (L.) Roxb)

\begin{tabular}{|c|c|c|c|c|c|c|}
\hline Sl. No & Character & Replication & Genotypes & Error & SEm \pm & CD@5\% \\
\hline Df & & $\mathbf{1}$ & 29 & 29 & & \\
\hline $\mathbf{A}$ & \multicolumn{6}{|l|}{ Growth parameters } \\
\hline 1. & Vine length 45 DAS & 3638.12 & $2208.48 * *$ & 490.87 & 22.15 & 45.31 \\
\hline 2. & Vine length 90DAS & 13081.00 & $14421.23 * *$ & 1085.74 & 32.95 & 67.39 \\
\hline 3. & Number of branches 90 DAS & 0.56 & $2.86^{* *}$ & 0.11 & 0.33 & 0.68 \\
\hline 4. & Number of leaves 45 DAS & 0.005 & $44.94 * *$ & 9.00 & 3.00 & 6.14 \\
\hline 5. & Number of leaves 90 DAS & 313.78 & $2652.61 * *$ & 77.68 & 8.81 & 18.03 \\
\hline B. & \multicolumn{6}{|l|}{ Earliness parameter: } \\
\hline 1. & Days to first female flower & 0.05 & $27.92 * *$ & 0.89 & 0.94 & 1.94 \\
\hline 2. & Days to first male flower & 0.22 & $10.91 * *$ & 0.67 & 0.82 & 1.68 \\
\hline 3. & Node to first female flower & 2.02 & $32.40^{* *}$ & 0.35 & 0.59 & 1.22 \\
\hline 4. & Node to first male flower & 0.17 & $0.25^{*}$ & 0.02 & 0.13 & 0.28 \\
\hline 5. & Days to $50 \%$ flowering & 0.06 & $9.10 * *$ & 0.68 & 0.82 & 1.70 \\
\hline 6. & Days to first harvest & 0.61 & $10.79 * *$ & 1.36 & 1.16 & 2.39 \\
\hline 7. & Days to last harvest & 6.62 & $53.83^{* *}$ & 3.68 & 1.91 & 3.93 \\
\hline 8. & Sex ratio & 0.10 & $45.46 * *$ & 0.08 & 0.28 & 0.58 \\
\hline C. & \multicolumn{6}{|l|}{ Yield and yield components: } \\
\hline 1 & Number of fruits per plant & 2.48 & $1.52^{* *}$ & 0.07 & 0.26 & 0.55 \\
\hline 2. & Fruit yield per plant & 49070.00 & $342163.72 * *$ & 3069.86 & 55.40 & 133.30 \\
\hline 3. & Fruit yield per plot & 2.17 & $30.02 * *$ & 0.13 & 0.36 & 0.74 \\
\hline 4. & Average fruit weight & 15.50 & $5972.36 * *$ & 22.45 & 4.73 & 9.69 \\
\hline 5. & Fruit length & 4.64 & $63.05 * *$ & 0.38 & 0.62 & 1.28 \\
\hline 6. & Fruit diameter & 98.98 & $53.91 * *$ & 2.58 & 1.60 & 3.25 \\
\hline & & & & significa & & \\
\hline
\end{tabular}


Table. 2 Genotypic correlation co-efficient among growth, earliness, yield and yield components in ridge gourd (Luffa acutangula (L.) Roxb.)

\begin{tabular}{|c|c|c|c|c|c|c|c|c|c|c|c|c|c|c|c|c|c|}
\hline & 2 & 3 & 4 & 5 & 6 & 7 & 8 & 9 & 10 & 11 & 12 & 13 & 14 & 15 & 16 & 17 & 18 \\
\hline 1 & $0.838 * *$ & $0.695 * *$ & $0.505 * *$ & $0.686 * *$ & -0.062 & -0.065 & $0.650 * *$ & $0.609 * *$ & -0.116 & -0.202 & $0.526 * *$ & $0.546^{* *}$ & $0.627 * *$ & $0.616 * *$ & $0.654 * *$ & $0.654 * *$ & $0.606^{* *}$ \\
\hline 2 & 1.000 & $0.860 * *$ & $0.669 * *$ & $0.880 * *$ & -0.224 & -0.272 & $0.690 * *$ & $0.647 * *$ & -0.265 & -0.301 & $0.746 * *$ & $0.770 * *$ & $0.805 * *$ & $0.774 * *$ & $0.790 * *$ & $0.764 * *$ & $0.713 * *$ \\
\hline 3 & & 1.000 & $0.645^{* *}$ & $0.775^{* *}$ & -0.310 & -0.309 & $0.756^{* *}$ & $0.636 * *$ & -0.320 & $-0.402 *$ & $0.783^{* *}$ & $0.649 * *$ & $0.703 * *$ & $0.708 * *$ & $0.717 * *$ & $0.722 * *$ & $0.730 * *$ \\
\hline 4 & & & 1.000 & $0.709 * *$ & $-0.484 * *$ & -0.346 & $0.660 * *$ & $0.458^{*}$ & $-0.407 *$ & $-0.377 *$ & $0.608 * *$ & $0.494 * *$ & $0.596 * *$ & $0.692 * *$ & $0.670 * *$ & $0.660 * *$ & $0.650 * *$ \\
\hline 5 & & & & 1.000 & $-0.579 * *$ & $-0.565 * *$ & $0.869 * *$ & $0.831 * *$ & $-0.503 * *$ & $-0.655^{* *}$ & $0.772 * *$ & $0.634 * *$ & $0.789 * *$ & $0.795 * *$ & $0.808 * *$ & $0.805^{* *}$ & $0.753^{* *}$ \\
\hline 6 & & & & & 1.000 & $0.884 * *$ & $0.898 * *$ & $0.784 * *$ & $0.882 * *$ & $0.913 * *$ & $-0.365^{*}$ & -0.253 & $-0.575^{* *}$ & $-0.690 * *$ & $-0.651 * *$ & $-0.662 * *$ & $-0.691 * *$ \\
\hline 7 & & & & & & 1.000 & $0.852 * *$ & $0.774 * *$ & $0.889 * *$ & $0.829 * *$ & $-0.358^{*}$ & -0.321 & $-0.472 * *$ & $-0.611 * *$ & $-0.571 * *$ & $-0.633 * *$ & $-0.589 * *$ \\
\hline 8 & & & & & & & 1.000 & $0.867 * *$ & $0.817 * *$ & $0.813 * *$ & $0.597 * *$ & $0.478 * *$ & $0.604 * *$ & $0.619 * *$ & $0.675 * *$ & $0.622 * *$ & $0.580^{* *}$ \\
\hline 9 & & & & & & & & 1.000 & $0.774 * *$ & $0.660 * *$ & $0.463 * *$ & 0.334 & $0.601 * *$ & $0.588 * *$ & $0.630 * *$ & $0.609 * *$ & $0.509 * *$ \\
\hline 10 & & & & & & & & & 1.000 & $0.848 * *$ & $-0.365^{*}$ & -0.273 & $-0.612 * *$ & $-0.684 * *$ & $-0.659 * *$ & $-0.637 * *$ & $-0.643 * *$ \\
\hline 11 & & & & & & & & & & 1.000 & $-0.362 *$ & -0.227 & $-0.589 * *$ & $-0.612 * *$ & $-0.580 * *$ & $-0.579 * *$ & $-0.520 * *$ \\
\hline 12 & & & & & & & & & & & 1.000 & $0.714 * *$ & $0.675 * *$ & $0.717 * *$ & $0.727 * *$ & $0.651 * *$ & $0.780 * *$ \\
\hline 13 & & & & & & & & & & & & 1.000 & $0.647 * *$ & $0.560 * *$ & $0.555^{* *}$ & $0.439 *$ & $0.531 * *$ \\
\hline 14 & & & & & & & & & & & & & 1.00 & $0.889 * *$ & $0.857 * *$ & $0.826 * *$ & $0.637 * *$ \\
\hline 15 & & & & & & & & & & & & & & 1.000 & $0.821 * *$ & $0.871 * *$ & $0.795^{* *}$ \\
\hline 16 & & & & & & & & & & & & & & & 1.000 & $0.959 * *$ & $0.781 * *$ \\
\hline 17 & & & & & & & & & & & & & & & & 1.000 & $0.810^{* *}$ \\
\hline 18 & & & & & & & & & & & & & & & & & 1.000 \\
\hline
\end{tabular}

\section{Critical $r$ value $=0.463$ at 1 per cent and 0.361 at 5 per cent}

* and ** indicate significant at 5 and 1 per cent probability level, respectively.

1. Vine length 45 DAS

2. Vine length 90 DAS

3. Number of branches 90 DAS

4. Number of leaves 45 DAS

5. Number of leaves 90 DAS

6. Days to first female flowering
7. Days to first male flowering

8. Node to first female flowering

9. Node to first male flowering

10. Days to $50 \%$ flowering

11. Days to first harvest

12. Days to last harvest
13. Sex ratio

14. Number of fruits per plant

15. Fruit yield per plant

16. |Fruit yield per plot

17. Average fruit weight

18. Fruit length 
Table.3 Phenotypic correlation co-efficient among growth, earliness, yield and yield components in ridge gourd (Luffa acutangula (L.) Roxb)

\begin{tabular}{|c|c|c|c|c|c|c|c|c|c|c|c|c|c|c|c|c|c|}
\hline & 2 & 3 & 4 & 5 & 6 & 7 & 8 & 9 & 10 & 11 & 12 & 13 & 14 & 15 & 16 & 17 & 18 \\
\hline 1 & $0.639 * *$ & $0.636 * *$ & $0.498 * *$ & $0.652^{* *}$ & -0.020 & -0.010 & $0.624 * *$ & $0.520^{* *}$ & -0.102 & -0.193 & $0.497 * *$ & $0.510^{* *}$ & $0.510^{* *}$ & $0.517^{* *}$ & $0.509^{* *}$ & $0.584 * *$ & $0.492 * *$ \\
\hline 2 & 1.000 & $0.702 * *$ & $0.535 * *$ & $0.734 * *$ & -0.210 & -0.205 & $0.650 * *$ & $0.560^{* *}$ & -0.211 & -0.226 & $0.659 * *$ & $0.709 * *$ & $0.704 * *$ & $0.760 * *$ & $0.746 * *$ & $0.707 * *$ & $0.663 * *$ \\
\hline 3 & & 1.000 & $0.663^{* *}$ & $0.756^{* *}$ & -0.265 & -0.300 & $0.734 * *$ & $0.577 * *$ & -0.317 & $-0.395^{*}$ & $0.692 * *$ & $0.619 * *$ & $0.607 * *$ & $0.650 * *$ & $0.657^{* *}$ & $0.686^{* *}$ & $0.608^{* *}$ \\
\hline 4 & & & 1.000 & $0.687 * *$ & $-0.460^{* *}$ & $-0.368^{*}$ & $0.538^{* *}$ & 0.314 & -0.357 & $-0.375^{*}$ & $0.513 * *$ & $0.407 *$ & $0.525 * *$ & $0.599 * *$ & $0.525 * *$ & $0.515^{* *}$ & $0.536 * *$ \\
\hline 5 & & & & 1.000 & $-0.550^{* * *}$ & $-0.527 * *$ & $0.844 * *$ & $0.737 * *$ & $-0.45^{* *}$ & $-0.449 * *$ & 0.681 ** & $0.615^{* *}$ & $0.762 * *$ & $0.768 * *$ & $0.766^{* *}$ & $0.715^{* *}$ & $0.731 * *$ \\
\hline 6 & & & & & 1.000 & $0.859 * *$ & $0.865^{* *}$ & $0.704 * *$ & $0.844 * *$ & $0.778^{* *}$ & -0.360 & -0.231 & $-0.553^{* *}$ & $-0.657 * *$ & $-0.631 * *$ & $-0.638^{* *}$ & $-0.671 * *$ \\
\hline 7 & & & & & & 1.000 & $0.797 * *$ & $0.675^{* *}$ & $0.845 * *$ & $0.769 * *$ & -0.357 & -0.304 & $-0.451^{*}$ & $-0.564 * *$ & $-0.535 * *$ & $-0.594^{* *}$ & $-0.557 * *$ \\
\hline 8 & & & & & & & 1.000 & $0.791 * *$ & $0.755^{* *}$ & $0.718 * *$ & $0.555^{* *}$ & $0.470 * *$ & $0.557 * *$ & $0.601 * *$ & $0.668^{* *}$ & $0.598 * *$ & $0.570 * *$ \\
\hline 9 & & & & & & & & 1.000 & $0.679 * *$ & $0.656^{* *}$ & $0.383 *$ & 0.316 & $0.527 * *$ & $0.557 * *$ & $0.612 * *$ & $0.579 * *$ & $0.506^{* *}$ \\
\hline 10 & & & & & & & & & 1.000 & $0.674^{* *}$ & $-0.362^{*}$ & -0.213 & $-0.548^{* * *}$ & $-0.627 * *$ & $-0.616^{* * *}$ & $-0.585^{* *}$ & $-0.600^{* *}$ \\
\hline 11 & & & & & & & & & & 1.000 & -0.358 & -0.190 & $-0.499 * *$ & $-0.537 * *$ & $-0.506^{* *}$ & $-0.505^{* *}$ & $-0.463 * *$ \\
\hline 12 & & & & & & & & & & & 1.000 & $0.708^{* *}$ & $0.516^{* *}$ & $0.682 * *$ & $0.693^{* *}$ & $0.608^{* *}$ & $0.732 * *$ \\
\hline 13 & & & & & & & & & & & & 1.000 & $0.618 * *$ & $0.554 * *$ & $0.553^{* *}$ & $0.437^{*}$ & $0.528 * *$ \\
\hline 14 & & & & & & & & & & & & & 1.000 & $0.862 * *$ & $0.811^{* *}$ & $0.781^{* *}$ & $0.608 * *$ \\
\hline 15 & & & & & & & & & & & & & & 1.000 & $0.807 * *$ & $0.858^{* *}$ & $0.784^{* *}$ \\
\hline 16 & & & & & & & & & & & & & & & 1.000 & $0.850^{* *}$ & $0.775^{* *}$ \\
\hline 17 & & & & & & & & & & & & & & & & 1.000 & $0.802 * *$ \\
\hline 18 & & & & & & & & & & & & & & & & & 1.000 \\
\hline
\end{tabular}

\section{Critical $r$ value $=0.463$ at 1 per cent and 0.361 at 5 per cent}

* and ** indicate significant at 5 and 1 per cent probability level, respectively.
1. Vine length 45 DAS
7. Days to first male flowering
2. Vine length $90 \mathrm{DAS}$
8. Node to first female flowering
3. Number of branches $90 \mathrm{DAS}$
4. Number of leaves 45 DAS
9. Node to first male flowering
5. Number of leaves 90 DAS
10. Days to $50 \%$ flowering
11. Days to first harvest
12. Days to last harvest
6. Days to first female flowering
13. Sex ratio
14. Number of fruits per plant
15. Fruit yield per plant
16. Fruit yield per plot
17. Average fruit weight
18. Fruit length 
Table.4 Genotypic path co-efficient analysis among growth, earliness, yield and yield component in ridge gourd (Luffa acutangula (L.) Roxb)

\begin{tabular}{|c|c|c|c|c|c|c|c|c|c|c|c|c|c|c|c|c|c|c|c|}
\hline & 1 & 2 & 3 & 4 & 5 & 6 & 7 & 8 & 9 & 10 & 11 & 12 & 13 & 14 & 15 & 16 & 17 & 18 & rG \\
\hline 1 & 0.066 & -0.152 & 0.102 & -0.037 & 0.208 & -0.174 & -0.075 & -0.033 & 0.108 & -0.110 & -0.172 & 0.173 & -0.047 & 0.273 & 0.201 & 0.217 & 0.018 & 0.050 & $0.616 * *$ \\
\hline 2 & 0.039 & -0.12 & 0.106 & -0.031 & 0.225 & -0.224 & -0.077 & -0.043 & 0.113 & -0.101 & -0.205 & 0.194 & -0.056 & 0.35 & 0.238 & 0.255 & 0.019 & 0.092 & $0.774 * *$ \\
\hline 3 & 0.032 & -0.161 & 0.112 & -0.041 & 0.223 & -0.292 & -0.079 & -0.035 & 0.167 & -0.118 & -0.379 & 0.230 & -0.055 & 0.387 & 0.253 & 0.278 & 0.110 & 0.076 & $0.708 * *$ \\
\hline 4 & 0.012 & -0.139 & 0.108 & -0.055 & 0.203 & -0.307 & -0.068 & -0.025 & 0.102 & -0.103 & -0.274 & 0.184 & -0.036 & 0.469 & 0.248 & 0.28 & 0.018 & 0.075 & $0.692 * *$ \\
\hline 5 & 0.047 & -0.170 & 0.125 & -0.039 & 0.283 & -0.382 & -0.097 & -0.037 & 0.149 & -0.143 & -0.353 & 0.201 & -0.046 & 0.513 & 0.348 & 0.292 & 0.019 & 0.085 & $0.795 * *$ \\
\hline 6 & 0.010 & -0.161 & 0.106 & -0.03 & 0.107 & -0.481 & -0.024 & -0.078 & 0.129 & -0.169 & -0.383 & 0.106 & -0.033 & 0.066 & 0.045 & 0.044 & 0.009 & 0.047 & $-0.690 * *$ \\
\hline 7 & 0.011 & -0.168 & 0.114 & -0.018 & 0.102 & -0.474 & -0.01 & -0.064 & 0.125 & -0.159 & & 0.105 & -0.024 & 0.064 & 0.043 & 0.043 & 0.009 & 0.053 & $-0.611 * *$ \\
\hline 8 & 0.024 & -0.115 & 0.172 & -0.037 & 0.220 & -0.489 & -0.012 & -0.010 & 0.164 & -0.146 & -0.405 & 0.155 & -0.035 & 0.497 & 0.275 & 0.282 & 0.010 & 0.069 & $0.619 * *$ \\
\hline 9 & 0.018 & -0.114 & 0.117 & -0.025 & 0.202 & -0.424 & -0.078 & -0.069 & 0.119 & -0.138 & -0.386 & 0.145 & -0.024 & 0.472 & 0.348 & 0.352 & 0.018 & 0.055 & $0.588 * *$ \\
\hline 10 & 0.020 & -0.138 & 0.108 & -0.026 & 0.108 & -0.492 & -0.01 & -0.071 & 0.112 & -0.178 & -0.402 & 0.102 & -0.035 & 0.068 & 0.046 & 0.047 & 0.008 & 0.049 & $-0.684 * *$ \\
\hline 11 & 0.010 & -0.160 & 0.103 & -0.026 & 0.102 & -0.418 & -0.014 & -0.052 & 0.109 & -0.151 & -0.375 & 0.101 & -0.039 & 0.061 & 0.038 & 0.042 & 0.009 & 0.048 & $-0.612 * *$ \\
\hline 12 & 0.040 & -0.114 & 0.135 & -0.039 & 0.273 & -0.354 & -0.065 & -0.065 & 0.194 & -0.134 & -0.326 & 0.160 & -0.057 & 0.450 & 0.271 & 0.28 & 0.011 & 0.057 & $0.717 * *$ \\
\hline 13 & 0.070 & -0.123 & 0.163 & -0.027 & 0.206 & -0.324 & -0.056 & -0.058 & 0.140 & -0.124 & -0.312 & 0.101 & -0.073 & 0.417 & 0.239 & 0.257 & 0.018 & 0.046 & $0.560 * *$ \\
\hline 14 & 0.054 & -0.18 & 0.161 & -0.038 & 0.222 & -0.285 & -0.065 & -0.018 & 0.114 & -0.109 & -0.251 & 0.176 & -0.047 & 0.486 & 0.26 & 0.306 & 0.017 & 0.086 & $0.889 * *$ \\
\hline 15 & 0.042 & -0.132 & 0.141 & -0.048 & 0.229 & -0.364 & -0.081 & -0.04 & 0.176 & -0.118 & -0.34 & 0.195 & -0.041 & 0.490 & 0.293 & 0.304 & 0.012 & 0.103 & $0.821 * *$ \\
\hline 16 & 0.041 & -0.121 & 0.157 & -0.042 & 0.218 & -0.378 & -0.082 & -0.052 & 0.106 & -0.114 & -0.303 & 0.189 & -0.032 & 0.555 & 0.297 & 0.318 & 0.011 & 0.103 & $0.871 * *$ \\
\hline 17 & 0.010 & -0.100 & 0.167 & -0.036 & 0.264 & -0.391 & -0.086 & -0.042 & 0.155 & -0.115 & -0.322 & 0.203 & -0.039 & 0.355 & 0.430 & 0.284 & 0.012 & 0.046 & $0.795 * *$ \\
\hline 18 & 0.038 & -0.139 & 0.177 & -0.029 & 0.203 & -0.388 & -0.042 & -0.052 & 0.155 & -0.143 & -0.339 & 0.167 & -0.024 & 0.337 & 0.359 & 0.292 & 0.014 & 0.140 & $0.726 * *$ \\
\hline
\end{tabular}

\section{Residual=0.0007. Diagonal values indicates direct effect $\quad \mathbf{r G}=$ Genotypic correlation coefficient of fruit yield per vine}

* and ** indicate significant at 5 and 1 per cent probability level, respectively.

1. Vine length 45 DAS

7. Days to first male flowering

2. Vine length 90 DAS

8. Node to first female flowering

3. Number of branches 90 DAS

4. Number of leaves 45 DAS

5. Number of leaves 90 DAS

6. Days to first female flowering
9. Node to first male flowering

10. Days to $50 \%$ flowering

11. Days to first harvest

12. Days to last harvest
13. Sex ratio

14. Number of fruits per plant

15. Fruit yield per plot

16. Average fruit weight

17. Fruit length

18. Fruit diameter 
Table.5 Phenotypic path co-efficient analysis among growth, earliness, yield and yield component in ridge gourd (Luffa acutangula (L.) Roxb)

\begin{tabular}{|c|c|c|c|c|c|c|c|c|c|c|c|c|c|c|c|c|c|c|c|}
\hline & 1 & 2 & 3 & 4 & 5 & 6 & 7 & 8 & 9 & 10 & 11 & 12 & 13 & 14 & 15 & 16 & 17 & 18 & $\mathbf{r P}$ \\
\hline 1 & -0.005 & -0.024 & 0.050 & -0.026 & 0.021 & -0.062 & -0.014 & 0.047 & 0.005 & -0.008 & -0.035 & 0.001 & 0.011 & 0.172 & 0.161 & 0.169 & 0.033 & 0.021 & $0.517 * *$ \\
\hline 2 & -0.004 & -0.029 & 0.061 & -0.023 & 0.024 & -0.066 & -0.015 & 0.054 & 0.006 & -0.008 & -0.047 & 0.001 & 0.015 & 0.265 & 0.207 & 0.258 & 0.044 & 0.017 & $0.760 * *$ \\
\hline 3 & -0.003 & -0.023 & 0.076 & -0.028 & 0.024 & -0.053 & -0.017 & 0.056 & 0.006 & -0.009 & -0.036 & 0.001 & 0.015 & 0.201 & 0.176 & 0.192 & 0.054 & 0.018 & $0.650 * *$ \\
\hline 4 & -0.003 & -0.026 & 0.05 & -0.033 & 0.019 & -0.042 & -0.011 & 0.041 & 0.003 & -0.007 & -0.034 & 0.001 & 0.009 & 0.206 & 0.174 & 0.202 & 0.036 & 0.014 & $0.599 * *$ \\
\hline 5 & -0.004 & -0.029 & 0.065 & -0.031 & 0.028 & -0.064 & -0.022 & 0.064 & 0.007 & -0.012 & -0.039 & 0.001 & 0.013 & 0.267 & 0.205 & 0.251 & 0.048 & 0.02 & $0.768 * *$ \\
\hline 6 & -0.002 & -0.094 & 0.02 & -0.092 & 0.021 & -0.216 & -0.091 & 0.026 & 0.007 & -0.173 & -0.192 & 0.001 & 0.009 & 0.038 & 0.028 & 0.032 & 0.01 & 0.011 & $-0.657 * *$ \\
\hline 7 & -0.002 & -0.078 & 0.011 & -0.071 & 0.018 & -0.195 & -0.076 & 0.021 & 0.006 & -0.159 & -0.172 & 0.002 & 0.007 & 0.037 & 0.026 & 0.037 & 0.012 & 0.012 & $-0.564 * *$ \\
\hline 8 & -0.003 & -0.021 & 0.056 & -0.023 & 0.024 & -0.072 & -0.048 & 0.076 & 0.008 & -0.022 & -0.012 & 0.001 & 0.01 & 0.207 & 0.164 & 0.188 & 0.051 & 0.017 & $0.601 * *$ \\
\hline 9 & -0.003 & -0.019 & 0.044 & -0.013 & 0.021 & -0.063 & -0.044 & 0.06 & 0.010 & -0.011 & -0.015 & 0.001 & 0.007 & 0.192 & 0.149 & 0.171 & 0.048 & 0.022 & $0.557 * *$ \\
\hline 10 & -0.002 & -0.094 & 0.026 & -0.094 & 0.021 & -0.213 & -0.092 & 0.027 & 0.007 & -0.176 & -0.189 & 0.003 & 0.01 & 0.032 & 0.029 & 0.027 & 0.04 & 0.011 & $-0.627 * *$ \\
\hline 11 & -0.002 & -0.075 & 0.022 & -0.073 & 0.018 & -0.198 & -0.057 & 0.024 & 0.006 & -0.151 & -0.182 & 0.005 & 0.01 & 0.029 & 0.022 & 0.024 & 0.031 & 0.01 & $-0.537 * *$ \\
\hline 12 & -0.002 & -0.019 & 0.060 & -0.022 & 0.019 & -0.062 & -0.015 & 0.042 & 0.004 & -0.009 & -0.021 & 0.001 & 0.015 & 0.233 & 0.189 & 0.205 & 0.049 & 0.015 & $0.682 * *$ \\
\hline 13 & -0.002 & -0.021 & 0.055 & -0.017 & 0.017 & -0.064 & -0.011 & 0.036 & 0.003 & -0.007 & -0.021 & 0.001 & 0.021 & 0.186 & 0.159 & 0.172 & 0.035 & 0.012 & $0.554 * *$ \\
\hline 14 & -0.003 & -0.013 & 0.061 & -0.012 & 0.022 & -0.053 & -0.016 & 0.068 & 0.006 & -0.009 & -0.02 & 0.001 & 0.023 & 0.273 & 0.235 & 0.223 & 0.049 & 0.027 & $0.862 * *$ \\
\hline 15 & -0.003 & -0.012 & 0.065 & -0.011 & 0.025 & -0.052 & -0.019 & 0.058 & 0.006 & -0.01 & -0.027 & 0.002 & 0.013 & 0.242 & 0.222 & 0.231 & 0.051 & 0.026 & $0.807 * *$ \\
\hline 16 & -0.003 & -0.019 & 0.06 & -0.026 & 0.023 & -0.043 & -0.021 & 0.061 & 0.007 & -0.009 & -0.021 & 0.001 & 0.012 & 0.262 & 0.243 & 0.251 & 0.053 & 0.027 & $0.858 * *$ \\
\hline 17 & -0.002 & -0.021 & 0.061 & -0.023 & 0.021 & -0.063 & -0.02 & 0.058 & 0.006 & -0.009 & -0.02 & 0.001 & 0.011 & 0.161 & 0.319 & 0.227 & 0.066 & 0.011 & $0.784 * *$ \\
\hline 18 & -0.003 & -0.013 & 0.067 & -0.016 & 0.025 & -0.074 & -0.011 & 0.064 & 0.003 & -0.009 & -0.024 & 0.001 & 0.007 & 0.159 & 0.245 & 0.204 & 0.02 & 0.038 & $0.683^{* *}$ \\
\hline
\end{tabular}

\section{Residual=0.0117. Diagonal values indicates direct effect $\quad \mathbf{r P}=\mathbf{G e n o t y p i c}$ correlation co-efficient of fruit yield per vine}

$*$ and ** indicate significant at 5 and 1 per cent probability level, respectively.

1. Vine length 45 DAS 7. Days to first male flowering

2. Vine length 90 DAS

3. Number of branches 90 DAS

4. Number of leaves 45 DAS

5. Number of leaves 90 DAS

6. Days to first female flowering
8. Node to first female flowering

9. Node to first male flowering

10. Days to $50 \%$ flowering

11. Days to first harvest

12. Days to last harvest

\author{
13. Sex ratio \\ 14. Number of fruits per plant \\ 15. Fruit yield per plot \\ 16. Average fruit weight \\ 17. Fruit length \\ 18. Fruit diameter
}


Similar results were obtained by Rao et al., (1999) in ridge gourd that high direct positive effect of number of fruits per vine on fruit yield, these results are in conformity with Prasanna et al., (2002) and Kantharaja (2003) in ridge gourd, Rathod (2007) in bitter gourd and Kumar et al., (2007) in bottle gourd. Therefore, direct selection for these traits would be rewarding for improvement of yield.

In conclusion, present study revealed that character like fruit yield per plant showed positive and significant correlation with vine length at 45 and 90 days after sowing, number of branches at 90 days after sowing, number of leaves at 45 and 90 days after sowing, node to first female and male flower, days to last harvest, sex ratio, number of fruits per plant, fruit yield per plant, fruit yield per plot and fruit yield per hectare. Since, the association is in desirable direction, selection for these traits may ultimately improve the yield. The number of fruits per plant, average fruit weight and fruit yield per plot were the most influencing factors. These characters have direct positive effect on fruit yield. Thus, these characters deserve greater weightage during selection for yield. The direct selection in these traits would be rewarding for improvement in the fruit yield per plant.

\section{References}

Al-Jibouri, H.A., Miller, P.A., and Robinson, H.V. 1958. Genotypic and environmental variance and covariances in an upland cotton cross of interspecific origin. Agron Journal, 50: 633-636.

Anonymous, 2008. Improved Cultivation Practices for Horticultural Crops (Kannada). University of Agricultural Sciences, Dharwad, pp. 84-86.

Choudhary, B., and Thakur, M.R. 1965. Inheritance of sex forms in Luffa. Indian J. of Genetic and Plant Breeding,
25(2): 188-197.

Chowdhury, D., and Sarma, K.C. 2002. Studies on variability, heritability, genetic advance and correlations in ridge gourd (Luffa acutangula Roxb.). Hort. J., 15(3): 53-58.

Dewey, D.R., and Lu, K.H. 1957. A correlation and path coefficient analysis of components of wheat grass seed production. Agron. J., 51: 515-518.

Katharaja, N.M., 2003, Studies on heterosis and combining ability in ridge gourd (Luffa acutangula (L.) Roxb.). M.Sc. (Hort.) Thesis, University of Agricultural Sciences, Dharwad.

Kumar, S., Singh, R. and Pal, A.K. 2007. Genetic variability, heritability, genetic advance, correlation coefficient and path analysis in bottle gourd. Indian $\mathrm{J}$. Hort., 64(2): 163-168.

Prasanna, S.C., Krishnappa, K.S. and Reddy, N.S. 2002. Correlation and path coefficient analysis studies in ridge gourd. Current Research, University of Agricultural Sciences, Bangalore. 31(9/10): 150-152.

Rao, B.N., Rao, P.V and Reddy, B. M. M. 2000. Correlation and path analysis in the segregating population of ridge gourd (Luffa acutangula (Roxb.) L.) Crop Research., 20 (2): 338-342.

Rao, B.N., Rao, P.V., and Reddy, T.B. 1999. Correlation and path coefficient studies in ridge gourd (Luffa acutangula (L.) Roxb.). Int. J. Tropical Agriculture, 17(1-4): 119-124.

Rathod, V. 2007. Studies on genetic variability and molecular characterization of bitter gourd (Momordica charantia L) genotypes. M.Sc. (Hort.) Thesis, University of Agricultural Sciences, Bangalore.

Robinson, R.W., and Decker-Walters, D.S. 1997. Cucurbits. New York Cab International. Pp. 226.

Varalakshmi, B., Rao, P.V., and Reddy, Y.N. 
1995. Genetic variability and heritability in ridge gourd (Luffa acutangula). Indian J. of Agricultural
Sciences, 65 (8): 608-610.

Wright, S., 1921. Correlation and causation. J. of Agricultural Research, 20: 557-587.

\section{How to cite this article:}

Hanume Gowda, K. 2020. Correlation and Path Co-efficient Analysis in Ridge Gourd [Luffa acutangula (L.) Roxb.]. Int.J.Curr.Microbiol.App.Sci. 9(07): 2965-2974.

doi: https://doi.org/10.20546/ijcmas.2020.907.350 